LYCEN 9109

April 1991

Revised: June 1991

\title{
On the $q$-analogue of the hydrogen atom
}

\author{
M Kibler ${ }^{1}$ and T Négadi ${ }^{2}$ \\ ${ }^{1}$ Institut de Physique Nucléaire de Lyon, IN2P3-CNRS et Université Claude \\ Bernard, F-69622 Villeurbanne Cedex, France \\ ${ }^{2}$ Laboratoire de Physique Théorique, Institut des Sciences Exactes, Univer- \\ sité d'Oran, Es-Sénia, Oran, Algérie
}

\begin{abstract}
The discrete spectrum of a $q$-analogue of the hydrogen atom is obtained from a deformation of the Pauli equations. As an alternative, the spectrum is derived from a deformation of the four-dimensional oscillator arising in the application of the Kustaanheimo-Stiefel transformation to the hydrogen atom. A model of the $2 s-2 p$ Dirac shift is proposed in the context of $q$-deformations.
\end{abstract}

(published in Journal of Physics A: Mathematical and General 24 (1991) 5283-5289) 


\section{Introduction}

In recent years, a great deal of work has been devoted to an apparently new mathematical structure, the structure of quantum group, and its application to various fields of physics (statistical mechanics, conformal quantum field theory, etc.). Indeed, the structure of quantum group (or algebra) is connected to those of Hecke algebra and quasi-triangular Hopf algebra and certainly plays an important rôle in non-commutative geometry (Drinfel'd 1985, Jimbo 1985, Woronowicz 1987). Loosely speaking, the structure of quantum algebra corresponds to a deformation which can be characterised by a deformation parameter $q$, an arbitrary complex number in the most general case.

There is no universal significance of the parameter $q$ and the physicist is tempted to consider it as a phenomenological parameter, something like a curvature constant, to be adjusted to experimental data. The limiting situation $q=1$ corresponds to the flat case and gives back the results afforded by (ordinary) quantum mechanics. For example, when $q$ goes to 1 , the quantum algebra $s u_{q}(2)$, which defines a $q$-analogue of angular momentum, reduces to the Lie algebra $s u(2)$ of (ordinary) angular momentum. The algebra $s u(2)$ is used from atoms to quarks with some reasonable success and, therefore, the replacement of $s u(2)$ by $s u_{q}(2)$ with $q$ near to 1 should be appropriate for the description of fine structure effects. In the framework of this philosophy, we may expect to obtain a fine structure of the hydrogen atom from a deformation of its non-relativistic spectrum.

It seems interesting to investigate $q$-analogues of dynamical systems in view of their potential use as refined units for modeling physical systems, 
with the case $q-1=0$ corresponding to already known effects and the case $q-1 \approx 0$ to new effects (as for instance spectrum shift and spectrum splitting). In this vein, the $q$-analogue of the harmonic oscillator, as derived among others by Macfarlane (1989), Biedenharn (1989), Sun and $\mathrm{Fu}$ (1989), and Kulish and Reshetikhin (1989), represents a first important step ; further, the $q$-analogues of various coherent states introduced recently (Quesne 1991, Katriel and Solomon 1991) might be interesting in physical applications.

It is the purpose of the present work to introduce a $q$-analogue of another very simple dynamical system, viz, the three-dimensional hydrogen atom. We shall deal here with the (quantum mechanical) discrete spectrum of the hydrogen atom in $\mathbf{R}^{3}$ and shall obtain in $\S 2$ its $q$-analogue by passing from the dynamical invariance algebra $s o(4)=s u(2) \oplus s u(2)$ of the ordinary hydrogen atom system to its $q$-analogue $s u_{q}(2) \oplus s u_{q}(2)$. In $\S 3$, an alternative spectrum will be derived from the Kustaanheimo-Stiefel (KS) transformation (Kustaanheimo and Stiefel 1965) and applied to a phenomenological derivation of the $2 s-2 p$ fine structure splitting. We shall close this paper with some conclusions in $\S 4$ about the non-unicity of $q$-analogues and the relevance of the quantum algebra $s o_{q}(3,2)$ for the Wigner-Racah algebra of $S U_{q}(2)$.

\section{2. $q$-analogue of the Pauli equations}

Since we want to examine $s u_{q}(2) \oplus s u_{q}(2)$, we review, in a non-standard presentation, some basic facts about the quantum algebra $s u_{q}(2)$. Let $\mathcal{E}=$ $\{\mid j m>: 2 j \in \mathbf{N}, m=-j(1) j\}$ be the Hilbert space of the representation theory of the (ordinary) Lie group $S U(2)$. We define the operators $a_{+}, a_{+}^{+}$, 
$a_{-}$and $a_{-}^{+}$acting on $\mathcal{E}$ by the relations

$$
\begin{gathered}
a_{ \pm}|j m>=\sqrt{[j \pm m]}| j-\frac{1}{2}, m \mp \frac{1}{2}> \\
a_{ \pm}^{+}|j m>=\sqrt{[j \pm m+1]}| j+\frac{1}{2}, m \pm \frac{1}{2}>.
\end{gathered}
$$

In equation $(1)$, we use the notation $[x] \equiv[x]_{q}$ where

$$
[x]_{q}=\frac{q^{x}-q^{-x}}{q-q^{-1}}
$$

is the $q$-analogue of the (real) number $x$; in this paper, $q$ is taken as a complex number which is not a root of unity. In the limiting situation $q=$ 1, equation (1) gives back the defining relations introduced by Schwinger (1952) in his theory of angular momentum (see also Kibler and Grenet (1980) and Nomura (1990)). Some trivial properties (matrix elements, timereversal behavior, adjointness relations, etc.) for the operators $a_{+}, a_{+}^{+}, a_{-}$ and $a_{-}^{+}$can be derived from the starting relations (1).

By introducing

$n_{1}=j+m \quad n_{2}=j-m \quad|j m>\equiv| j+m, j-m>=\mid n_{1}, n_{2}>$

we immediately obtain

$a_{+}\left|n_{1} n_{2}>=\sqrt{\left[n_{1}\right]}\right| n_{1}-1, n_{2}>\quad a_{+}^{+}\left|n_{1} n_{2}>=\sqrt{\left[n_{1}+1\right]}\right| n_{1}+1, n_{2}>$

$a_{-}\left|n_{1} n_{2}>=\sqrt{\left[n_{2}\right]}\right| n_{1}, n_{2}-1>\quad a_{-}^{+}\left|n_{1}, n_{2}>=\sqrt{\left[n_{2}+1\right]}\right| n_{1}, n_{2}+1>$. 
Consequently, the sets $\left\{a_{+}, a_{+}^{+}\right\}$and $\left\{a_{-}, a_{-}^{+}\right\}$are two commuting sets of $q$-bosons. Indeed, these $q$-bosons satisfy the relations

$$
\begin{gathered}
a_{+} a_{+}^{+}-q^{-1} a_{+}^{+} a_{+}=q^{N_{1}} \quad a_{-} a_{-}^{+}-q^{-1} a_{-}^{+} a_{-}=q^{N_{2}} \\
{\left[a_{+}, a_{-}\right]_{-}=\left[a_{+}^{+}, a_{-}^{+}\right]_{-}=\left[a_{+}, a_{-}^{+}\right]_{-}=\left[a_{+}^{+}, a_{-}\right]_{-}=0}
\end{gathered}
$$

where $N_{1}$ and $N_{2}$ are the (usual) number operators defined by

$$
N_{1}\left|n_{1} n_{2}>=n_{1}\right| n_{1} n_{2}>\quad N_{2}\left|n_{1} n_{2}>=n_{2}\right| n_{1} n_{2}>.
$$

Equations (4-6) show that each of the sets $\left\{a_{+}, a_{+}^{+}\right\}$and $\left\{a_{-}, a_{-}^{+}\right\}$is a set of $q$-bosons as introduced independently by many authors including Macfarlane (1989), Biedenharn (1989), and Sun and Fu (1989).

We now introduce the three following operators

$$
J_{-}=a_{-}^{+} a_{+} \quad J_{3}=\frac{1}{2}\left(N_{1}-N_{2}\right) \quad J_{+}=a_{+}^{+} a_{-} .
$$

The action of $J_{-}, J_{3}$ and $J_{+}$on the space $\mathcal{E}$ is given by

$$
J_{3}|j m>=m| j m>\quad J_{ \pm}|j m>=\sqrt{[j \mp m][j \pm m+1]}| j, m \pm 1>.
$$

We thus recover the Jimbo representation of the quantum group $S U_{q}(2)$. The commutation relations of the operators $J_{-}, J_{3}$ and $J_{+}$easily follow from (8). As a matter of fact, we have

$$
\left[J_{3}, J_{ \pm}\right]_{-}= \pm J_{ \pm} \quad\left[J_{+}, J_{-}\right]_{-}=\left[2 J_{3}\right]
$$


In equation (9), the abbreviation $[A] \equiv[A]_{q}$ stands for the $q$-analogue

$$
[A]_{q}=\frac{q^{A}-q^{-A}}{q-q^{-1}}
$$

of the operator $A$ acting on $\mathcal{E}$. The relations (9) characterise the quantum algebra $s u_{q}(2)$.

A basic ingredient of $s u_{q}(2)$, of central importance for what follows, is its Casimir operator $J^{2}$. We shall take it in the form

$$
J^{2}=\frac{1}{2}\left(J_{+} J_{-}+J_{-} J_{+}\right)+\frac{[2]}{2}\left[J_{3}\right]^{2}
$$

so that the eigenvalues of $J^{2}$ on $\mathcal{E}$ are $[j][j+1]$ with $2 j$ in $\mathbf{N}$. The form taken in the present paper for $J^{2}$ differs from the one usually encountered in the literature ; indeed, equation (11) ensures that the eigenvalues of $J^{2}$ are merely $j(j+1)$ in the limiting case $q=1$, a result which turns out to be essential in the framework of the philosophy sketched in the introduction.

We are in a position to $q$-deform the Pauli equations for the threedimensional hydrogen atom in the case of the discrete spectrum. From the orbital angular momentum $\mathbf{L}$ and the Laplace-Runge-Lenz-Pauli vector $\mathbf{M}$ for the ordinary hydrogen atom system, we define the operators $\mathbf{A}$ and $\mathbf{B}$ through

$$
\mathbf{A}=\frac{1}{2}(\mathbf{L}+\mathbf{N}) \quad \mathbf{B}=\frac{1}{2}(\mathbf{L}-\mathbf{N}) \quad \mathbf{N}=\sqrt{\frac{-\mu}{2 E}} \mathbf{M}
$$

where $E$ is the energy $(E<0)$ and $\mu$ the reduced mass of the atom. We know that $\left\{A_{i}: i=1,2,3\right\}$ and $\left\{B_{i}: i=1,2,3\right\}$ are the generators of two groups, say $A S U(2)$ and $B S U(2)$, both of them being of the $S U(2)$ 
type. In addition, the Casimir operators $A^{2}$ and $B^{2}$ of the Lie algebras $a s u(2)$ and $b s u(2)$ satisfy the equations (see Pauli 1926)

$$
A^{2}-B^{2}=0 \quad E\left(2 A^{2}+2 B^{2}+\hbar^{2}\right)=-\frac{1}{2} \mu Z^{2} e^{4}
$$

valid for a hydrogenlike atom with a nuclear charge $Z e$. A possible way to define a $q$-deformed hydrogen atom is to deform the Lie groups $A S U(2)$ and $B S U(2)$. Then, the Pauli equations (13) have to be extended to the quantum algebra $a s u_{q}(2) \oplus b s u_{q}(2)$. This deformation produces a $q$-analogue of the hydrogen atom, the energy $E$ of which is given by

$$
E \equiv E_{j}=\frac{1}{4[j][j+1]+1} E_{0} \quad 2 j \in \mathbf{N}
$$

with

$$
E_{0}=-\frac{1}{2} \frac{\mu Z^{2} e^{4}}{\hbar^{2}}
$$

Equations (14) and (15) follow from (13) where we have introduced the eigenvalues of $A^{2}$ and $B^{2}$, in correct units, of the Casimir operators of $a s u_{q}(2)$ and $b s u_{q}(2)$.

The quantity $E_{0}$ is the energy of the ground state of the $q$-deformed hydrogen atom. The $q$-deformed hydrogen atom defined by (14) thus has the same ground energy level as the ordinary hydrogen atom which corresponds to the limiting situation $q=1$. Furthermore, its (discrete) spectrum exhibits the same degeneracy as that of the ordinary hydrogen atom. The only difference between the cases $q=1$ and $q \neq 1$ arises in the position of the excited levels. Of course, the $q$-deformed spectrum reduces to that of the ordinary hydrogen atom when $q$ goes to 1 ; the principal quantum number $n$ is then given by $n=2 j+1$. 


\section{3. q-analogue and $\mathrm{KS}$ transformation}

In the case $q=1$, we know how to pass from the hydrogen atom in $\mathbf{R}^{3}$ to an isotropic harmonic oscillator in $\mathbf{R}^{4}$ or $\mathbf{R}^{2} \otimes \mathbf{R}^{2}$ by applying the KS transformation (Boiteux 1972, Kibler and Négadi 1983a, b, 1984, Kibler, Ronveaux and Négadi 1986). The reader may consult Kustaanheimo and Stiefel (1965), Cornish (1984), Lambert and Kibler (1988), and Hage Hassan and Kibler (1991) for a description of this transformation, which is indeed a particular Hurwitz transformation. The KS transformation can be used to define a $q$-deformed hydrogen atom. This may be achieved along the following lines : (i) apply the KS transformation to the ordinary hydrogen atom in $\mathbf{R}^{3}$ in order to obtain an isotropic harmonic oscillator in $\mathbf{R}^{4}$, (ii) transpose the latter oscillator into its $q$-analogue and (iii) invoke the "inverse" KS transformation to get a $q$-analogue of the hydrogen atom.

As a result, we obtain a $q$-deformed hydrogen atom characterised by the discrete spectrum

$$
\begin{gathered}
E \equiv E_{n_{1} n_{2} n_{3} n_{4}}=\frac{16}{\nu\left(n_{1} n_{2} n_{3} n_{4}\right)^{2}} E_{0} \\
\nu\left(n_{1} n_{2} n_{3} n_{4}\right)=\sum_{i=1}^{4}\left(\left[n_{i}\right]+\left[n_{i}+1\right]\right) \quad n_{i} \in \mathbf{N} \quad(i=1,2,3,4) .
\end{gathered}
$$

In the limiting case $q=1$, the principal quantum number $n$ is connected to the oscillator quantum numbers $n_{i}(i=1,2,3,4)$ by $n_{1}+n_{2}+n_{3}+n_{4}+2=$ $2 n$. Then, the $q$-deformed spectrum (16) gives back the discrete spectrum of the ordinary hydrogen atom when $q$ goes to 1 . Here again, the $q$-deformed hydrogen atom has the same ground energy level as the ordinary hydrogen atom. However, we now pass from the discrete spectrum of the ordinary 
hydrogen atom to the one of this alternative $q$-deformed hydrogen atom by means of a level splitting for the excited levels.

As an illustration, the level corresponding to the principal quantum number $n=2$ when $q=1$ is split into two levels when $q \neq 1$. In fact, we have the two energy levels

$$
E_{2000}=\frac{16}{([2]+[3]+3)^{2}} E_{0} \quad E_{1100}=\frac{4}{([2]+2)^{2}} E_{0} .
$$

We thus have a splitting of the $n=2$ non-relativistic level, a situation which is reminiscent of the fine structure splitting afforded by the Dirac theory of the hydrogen atom. By using the oscillator basis $\left\{\Phi_{n_{1} n_{2} n_{3} n_{4}}\right\}$ described by Kibler, Ronveaux and Négadi (1986), it can be proved that the wave functions $\Psi_{n \ell m}$ corresponding to the limiting case $q=1$ are

$$
\begin{aligned}
& \Psi_{200}=N\left(\Phi_{2000}+\Phi_{0200}+\Phi_{0020}+\Phi_{0002}\right) \\
& \Psi_{210}=N\left(\Phi_{2000}+\Phi_{0200}-\Phi_{0020}-\Phi_{0002}\right)
\end{aligned}
$$

for (the doublet) $E_{2000}$, and

$$
\begin{aligned}
\Psi_{211} & =N\left[\Phi_{1010}-\Phi_{0101}+i\left(\Phi_{1001}-\Phi_{0110}\right)\right] \\
\Psi_{21-1} & =N\left[\Phi_{1010}-\Phi_{0101}-i\left(\Phi_{1001}-\Phi_{0110}\right)\right]
\end{aligned}
$$

for (the doublet) $E_{1100}$. Equation (17) illustrates the fact that, when going from $q=1$ to $q \neq 1$, the spectrum is not only shifted but also split (except for the ground energy level). From (17), we obtain the splitting

$$
\Delta_{q}=E_{1100}-E_{2000}=\frac{3}{16} E_{0}(q-1)^{2} \quad \text { for } \quad q-1 \approx 0 .
$$

We note that by taking $q=1.004$, we get $\Delta_{q}=0.33 \mathrm{~cm}^{-1}$. The so-obtained value for $\Delta_{q}$ has the order of magnitude of the $\left(2 p^{2} P_{\frac{3}{2}}\right)-\left(2 s^{2} S_{\frac{1}{2}}, 2 p^{2} P_{\frac{1}{2}}\right)$ experimental fine structure splitting. 


\section{Closing remarks}

The application of quantum groups to a large class of physical problems requires the $q$-deformation of the usual dynamical systems. In this direction, the $q$-deformation of the one-dimensional harmonic oscillator (Macfarlane 1989, Biedenharn 1989) has been a decisive step. The $q$-analogues of the hydrogen atom in $\mathbf{R}^{3}$ introduced in this work represent a further important step. Part of this work might be extended to the hydrogen atom in $\mathbf{R}^{5}$ by means of the Hurwitz transformation associated to the Hopf fibration on spheres $S^{7} \rightarrow S^{4}$ of compact fiber $S^{3}$ (Lambert and Kibler 1988, Hage Hassan and Kibler 1991).

The hydrogen atom in $\mathbf{R}^{3}$ is a basic dynamical system in classical and quantum mechanics in the case $q=1$. (Ordinary quantum mechanics can be thought to correspond to the limiting case $q=1$.) This system is maximally super-integrable (Evans 1990, Kibler and Winternitz 1990) with rich dynamical invariance and non-invariance algebras. It would be interesting to investigate invariance and non-invariance algebras of the $q$-deformed hydrogen atom and to see the branching rules when going from $q=1$ (ordinary quantum mechanics) to $q \neq 1$ ( $q$-deformed quantum mechanics). Similar remarks apply to the isotropic harmonic oscillator in $\mathbf{R}^{3}$, another maximally super-integrable system.

There are several ways to define a $q$-analogue of the hydrogen atom. We have been concerned in this article with two of them. There are other solutions connected to pairs of non-commuting $q$-bosons. The two $q$-deformed hydrogen atoms defined in $\S 3$ and $\S 4$ by their discrete spectra are different although having the same (ordinary) quantum limit (corresponding to 
$q=1$ ). This constitutes in fact a general problem we face when dealing with $q$-deformed objects (cf., for example, the case of coherent states introduced by Katriel and Solomon (1991)). We presently do not have a simple correspondance principle for associating a (unique) $q$-analogue to a given mathematical or physical object. The use of a clearly defined, via the notion of $q$-derivative, $q$-deformed Schrödinger equation should lead to some standardisation of $q$-deformed objects.

Among the two $q$-deformed hydrogen atoms discussed above, the one obtained via the $\mathrm{KS}$ transformation is certainly the most interesting. It leads to a spectrum which is shifted and split with respect to the ordinary quantum limit corresponding to $q=1$. Furthermore, the $2 s-2 p$ Dirac splitting can be reproduced by adjusting the value of $q$ and this yields a value close to 1 . The latter result should not be taken too seriously but it however constitutes a model of the $2 s-2 p$ splitting without relativistic quantum mechanics.

We close this section with a remark about the $q$-bosonisation (7) of the spherical angular momentum $\left\{J_{-}, J_{3}, J_{+}\right\}$leading to $s u_{q}(2)$. We may also define an hyperbolic angular momentum $\left\{K_{-}, K_{3}, K_{+}\right\}$by

$$
K_{-}=a_{+} a_{-} \quad K_{3}=\frac{1}{2}\left(N_{1}+N_{2}+1\right) \quad K_{+}=a_{+}^{+} a_{-}^{+}
$$

with the property

$$
\begin{gathered}
K_{-}|j m>=\sqrt{[j-m][j+m]}| j-1, m> \\
K_{3}\left|j m>=\left(j+\frac{1}{2}\right)\right| j m> \\
K_{+}|j m>=\sqrt{[j-m+1][j+m+1]}| j+1, m>.
\end{gathered}
$$


The operators $K_{-}, K_{3}$ and $K_{+}$satisfy the commutation relations

$$
\left[K_{3}, K_{ \pm}\right]_{-}= \pm K_{ \pm} \quad\left[K_{+}, K_{-}\right]_{-}=-\left[2 K_{3}\right]
$$

and thus span the quantum algebra $s u_{q}(1,1)$. There are four other bilinear forms of the $q$-bosons $a_{+}, a_{+}^{+}, a_{-}$and $a_{-}^{+}$which can be formed in addition to the $J$ 's and the $K$ 's, viz,

$$
k_{+}^{+}=-a_{+}^{+} a_{+}^{+} \quad k_{-}^{+}=a_{-}^{+} a_{-}^{+} \quad k_{-}^{-}=-a_{+} a_{+} \quad k_{+}^{-}=a_{-} a_{-} .
$$

The operators $k$ 's are clearly shift operators for the quantum numbers $j$

and $m$. It can be proved that the set $\left\{k_{+}^{+}, k_{-}^{+}, k_{-}^{-}, k_{+}^{-}\right\}$span, together with the sets $\left\{J_{-}, J_{3}, J_{+}\right\}$and $\left\{K_{-}, K_{3}, K_{+}\right\}$, a quantum algebra which may be identified to $s o(3,2) \sim s p(4, \mathbf{R})$ when $q$ goes to 1 . The quantum algebra $s o_{q}(3,2)$ might be useful for studying the Wigner-Racah algebra of the group $S U_{q}(2)$ as was done in the case $q=1$ (Kibler and Grenet 1980).

\section{Acknowledgments}

One of the authors (M K) thanks Y Saint-Aubin for communicating his lectures notes (Saint-Aubin 1990) on quantum groups. He is grateful to J Katriel, G Rideau and S L Woronowicz for interesting discusions. 


\section{References}

Biedenharn L C 1989 J. Phys. A: Math. Gen. 22 L873

Boiteux M 1972 C. R. Acad. Sci., Paris 274B 867

Cornish F H J 1984 J. Phys. A: Math. Gen. 172191

Drinfel'd V G 1985 Sov. Math. Dokl. 32254

Evans N W 1990 Phys. Lett. 147A 483

Hage Hassan M and Kibler M 1991 On Hurwitz transformations Report LYCEN 9110

Jimbo M 1985 Lett. Math. Phys. 1063

Katriel J and Solomon A I 1991 J. Phys. A: Math. Gen. 242093

Kibler M and Grenet G 1980 J. Math. Phys. 21422

Kibler M and Négadi T 1983a Lett. Nuovo Cimento 37225

— 1983b J. Phys. A: Math. Gen. 164265

— 1984 Phys. Rev. A 292891

Kibler M, Ronveaux A and Négadi T 1986 J. Math. Phys. 271541

Kibler M and Winternitz P 1990 Phys. Lett. 147A 338

Kulish P P and Reshetikhin N Yu 1989 Lett. Math. Phys. 18143 
Kustaanheimo P and Stiefel E 1965 J. Reine Angew. Math. 218204

Lambert D and Kibler M 1988 J. Phys. A: Math. Gen. 21307

Macfarlane A J 1989 J. Phys. A: Math. Gen. 224581

Nomura M 1990 J. Phys. Soc. Jpn. 592345

Pauli W 1926 Z. Phys. 36336

Quesne C 1991 Phys. Lett. 153A 303

Saint-Aubin Y 1990 Quantum groups and their application to conformal quantum field theories Report CRM-1663

Schwinger J 1952 On angular momentum Report U.S. AEC NYO-3071 (reprinted in 1965 Quantum Theory of Angular Momentum ed L C Biedenharn and H van Dam (New York: Academic) p 229)

Sun C-P and Fu H-C 1989 J. Phys. A: Math. Gen. 22 L983

Woronowicz S L 1987 Comm. Math. Phys. 111613 